1 Hacettepe Journal of Mathematics and Statistics

$\bigcap$ Volume $44(2)$ (2015), 331-339

\title{
Oscillation of fourth-order nonlinear neutral delay dynamic equations
}

\author{
S. R. Grace* and A. Zafer ${ }^{\ddagger}$
}

\begin{abstract}
In this work we establish some new sufficient conditions for oscillation of fourth-order nonlinear neutral delay dynamic equations of the form $\left(a(t)\left([x(t)-p(t) x(h(t))]^{\Delta \Delta \Delta}\right)^{\alpha}\right)^{\Delta}+q(t) x^{\beta}(g(t))=0, \quad t \in\left[t_{0}, \infty\right)_{\mathbb{T}}$,
\end{abstract} where $\alpha$ and $\beta$ are quotients of positive odd integers with $\beta \leq \alpha$.

2000 AMS Classification: 34C10, 34C15.

Keywords: Oscillation, Neutral, Time scales, Fourth-order.

Received 26 /01 /2012 : Accepted 27 /02 /2013 Doi : 10.15672/HJMS.2015449415

\section{Introduction}

In this work we investigate the oscillatory behaviour of solutions of fourth order halflinear and sub-half-linear neutral delay dynamic equations of the form

$$
\left(a(t)\left((x(t)-p(t) x(h(t)))^{\Delta \Delta \Delta}\right)^{\alpha}\right)^{\Delta}+q(t) x^{\beta}(g(t))=0, \quad t \in\left[t_{0}, \infty\right)_{\mathbb{T}}
$$

on an arbitrary time scale $\mathbb{T}$ with the property that $0 \leq t_{0} \in \mathbb{T}$ and $\sup \mathbb{T}=\infty$.

A time scale $\mathbb{T}$ is a nonempty closed subset of $\mathbb{R}$, introduced by Hilger [12] in order to unify the continuous and discrete analysis. By a time scale interval $\left[t_{*}, \infty\right)_{\mathbb{T}}$ it is meant $\left[t_{*}, \infty\right) \cap \mathbb{T}$.

We assume that the following conditions are satisfied:

(i) $\alpha, \beta$ are quotients of positive odd integers with $\beta \leq \alpha$,

${ }^{*}$ Department of Engineering Mathematics, Faculty of Engineering, Cairo University, Orman, Giza 12221, Egypt.

Email: srgrace@eng.cu.edu.eg

${ }^{\dagger}$ Department of Mathematics, Middle East Technical University, 06800 Ankara, Turkey. Email: zafer@metu.edu.tr

${ }^{\ddagger}$ Current address: Department of Mathematics and Statistics, College of Engineering and Technology, American University of the Middle East, Kuwait.

Email: agacik.zafer@aum.edu.kw 
(ii) $a:\left[t_{0}, \infty\right)_{\mathbb{T}} \rightarrow \mathbb{R}_{+}:=(0, \infty)$ satisfies $a^{\Delta}(t) \geq 0$ and

$$
\int_{t_{0}}^{\infty} a^{-1 / \alpha}(t) \Delta t=\infty
$$

(iii) $p, q:\left[t_{0}, \infty\right)_{\mathbb{T}} \rightarrow \mathbb{R}_{+}$are rd-continous,

(iv) $g, h: \mathbb{T} \rightarrow \mathbb{T}$ satisfy $g(t) \leq t, h(t) \leq t, g^{\Delta}(t)>0, h^{\Delta}(t)>0$, and $\lim _{t \rightarrow \infty} g(t)=$ $\lim _{t \rightarrow \infty} h(t)=\infty$

(v) $l(t):=h^{-1} \circ g(t)$ satisfies $l^{\Delta}(t) \geq 0$ and $\lim _{t \rightarrow \infty} l(t)=\infty$.

By a solution of (1.1), we mean a nontrivial at infinity function $x \in C_{r d}\left[t_{-1}, \infty\right)_{\mathbb{T}}$ that satisfies (1.1), where $t_{-1}=\inf \left\{g(t): t \geq t_{0}\right\} \cap \inf \left\{h(t): t \geq t_{0}\right\}$. We tacitly assume that (1.1) possesses such solutions. Recall that such a solution of Eq. (1.1) is called nonoscillatory if there exists a $t_{0}^{*} \geq t_{0}$ such that $x(t) x^{\sigma}(t)>0$ for all $t \in\left[t_{0}^{*}, \infty\right)_{\mathbb{T}}$; otherwise, it is said to be oscillatory. Eq. (1.1) is oscillatory if all its solutions are oscillatory.

We note that there is an extensive study concerning the oscillation of second-order dynamic equations on time scales $[1,3,7,11,14]$. For some works on oscillation and asymptotic behaviour of third-order dynamic equations, see [5, 10]. Fourth-order dynamic equations are rarely considered in the literature due to difficulties peculiar to such equations $[8,13]$. Our aim in this paper is to make a contribution to the oscillation of fourth-order equations of the form (1.1).

\section{Preliminaries}

To start, we first provide some notations of the time scale calculus to be used in this work. For more details we refer the reader to [3].

2.1. Definition. Let $\mathbb{T}$ be a time scale and $t \in \mathbb{T}$. The forward and backward jump operators $\sigma, \rho: \mathbb{T} \rightarrow \mathbb{T}$ are defined by $\sigma(t):=\inf \{s \in \mathbb{T}: s>t\}$ and $\rho(t):=\sup \{s \in \mathbb{T}$ : $s<t\}$.

2.2. Definition. A point $t \in \mathbb{T}$ with $t>\inf \mathbb{T}$ is called right-scattered, right-dense, leftscattered and left-dense if $\sigma(t)>t, \sigma(t)=t, \rho(t)<t$ and $\rho(t)=t$ holds, respectively. Points that are left-dense and right-dense at the same time are called dense. The set $\mathbb{T}^{\kappa}$ is derived from $\mathbb{T}$ as follows: If $\mathbb{T}$ has a left-scattered maximum $m$, then $\mathbb{T}^{\kappa}=\mathbb{T}-\{m\}$; otherwise, $\mathbb{T}^{\kappa}=\mathbb{T}$.

2.3. Definition. Let $f: \mathbb{T} \rightarrow \mathbb{R}$. The delta derivative of $f$ at $t \in \mathbb{T}^{\kappa}$, denoted by $f^{\Delta}(t)$, to be the number (provided it exists) with the property such that for every $\epsilon>0$, there exists a neighbourhood $\mathbb{U}$ of $t$ with

$$
\left|f^{\sigma}(t)-f(s)-f^{\Delta}(t)[\sigma(t)-s]\right| \leq \epsilon|\sigma(t)-s| \text { for all } s \in \mathbb{U},
$$

where and in the sequel $f^{\sigma}(t):=f(\sigma(t))$ is used.

2.4. Definition. A function $f: \mathbb{T} \rightarrow \mathbb{R}$ is called right-dense continuous (rd-continuous) if $f$ is continuous at right-dense points in $\mathbb{T}$ and its left-sided limit exists (finite) at left-dense points in $\mathbb{T}$.

Every rd-continuous function has an antiderivative. A function $F: \mathbb{T} \rightarrow \mathbb{R}$ is called an antiderivative of a function $f: \mathbb{T}^{\kappa} \rightarrow \mathbb{R}$ if $F^{\Delta}(t)=f(t)$ holds for all $t \in \mathbb{T}^{\kappa}$. In this case the integral of $f$ is defined by

$$
\int_{a}^{b} f(s) \Delta s=F(b)-F(a) .
$$


Riemann and Lebesque integrals on an arbitrary time scale as well as improper integrals are introduced in $[2,9]$.

2.5. Definition. Taylor monomials $h_{n}(t, s): \mathbb{T} \times \mathbb{T} \rightarrow \mathbb{R}$ are defined [3] by

$$
\begin{aligned}
& h_{n+1}(t, s)=\int_{s}^{t} h_{n}(\tau, s) \Delta \tau, \quad n=1,2, \ldots \\
& h_{0}(t, s)=1 .
\end{aligned}
$$

We need the following lemmas.

2.6. Lemma. Let (1.2) hold and

$$
0<p(t) \leq 1 \text { eventually. }
$$

If $x$ is an eventually positive solution of (1.1), then for $z(t)=x(t)-p(t) x(h(t))$ there are only the following three possibilities:

(a) $z(t)>0, z^{\Delta}(t)>0, z^{\Delta \Delta}(t)>0, z^{\Delta \Delta \Delta}(t)>0, z^{\Delta^{4}}(t)<0$;

(b) $z(t)>0, z^{\Delta}(t)>0, z^{\Delta \Delta}(t)<0, z^{\Delta \Delta \Delta}(t)>0, z^{\Delta^{4}}(t)<0$;

(c) $z(t)<0, z^{\Delta}(t)>0, z^{\Delta \Delta}(t)<0, z^{\Delta \Delta \Delta}(t)>0, z^{\Delta^{4}}(t)<0$

for $t$ sufficiently large.

Proof. We see from Eq. (1.1) that $\left(a(t)\left(z^{\Delta \Delta \Delta}\right)^{\alpha}\right)^{\Delta}<0$ eventually. So, $a\left(z^{\Delta \Delta \Delta}\right)^{\alpha}$ is eventually monotone. Suppose that $z^{\Delta \Delta \Delta}(t)<0$ for $t$ sufficiently large. By using (1.2) we see that $z^{\Delta \Delta}(t) \rightarrow-\infty$ as $t \rightarrow \infty$ and hence $z(t) \rightarrow-\infty$ as $t \rightarrow \infty$. In particular, we have

$$
x(t) \leq p(t) x(h(t)) \leq x(h(t))
$$

and so $x$ is bounded. But this implies that $z$ is also bounded, a contradiction.

Thus we must have $z^{\Delta \Delta \Delta}(t)>0$. Since $a^{\Delta}(t) \geq 0$, it follows from

$$
\left.\left(a(t)\left(z^{\Delta \Delta \Delta}\right)^{\alpha}\right)^{\Delta}=a^{\sigma}(t)\left(z^{\Delta \Delta \Delta}\right)^{\alpha}\right)^{\Delta}+a^{\Delta}(t)\left(z^{\Delta \Delta \Delta}\right)^{\alpha}
$$

that $\left(\left(z^{\Delta \Delta \Delta}(t)\right)^{\alpha}\right)^{\Delta}<0$ eventually. Now using the time scales chain rule [3] with $y=$ $z^{\Delta \Delta \Delta}$, we obtain

$$
\left(y^{\alpha}\right)^{\Delta}(t)=\alpha y^{\Delta}(t) \int_{0}^{1}\left(h y^{\sigma}(t)+(1-h) y(t)\right)^{\alpha-1} d h
$$

Since the integral is nonnegative, we have $y^{\Delta}(t)<0$, i.e.,

$$
z^{\Delta^{4}}(t)<0
$$

If $z(t)>0$, then in view of (2.2) it follows from Kiguradze's lemma, see [4, 6], that either (a) or (b) holds. In case $z(t)<0$, we see as above that $z(t)$ is bounded, and hence (c) holds.

2.7. Lemma. Let (1.2) hold and

$$
-1<p(t) \leq 0 \text { eventually. }
$$

If $x$ is an eventually positive solution of (1.1), then for $z(t)=x(t)-p(t) x(h(t))$ there are only two possibilities (a) and (b) of Lemma 2.6.

Proof. It suffices to note that $z(t)$ is eventually positive due to $z(t) \geq x(t)$. 
2.8. Lemma. [5, Lemma 4]. If a function y satisfies

$$
y(t)>0, y^{\Delta}(t)>0, y^{\Delta \Delta}(t)>0, y^{\Delta \Delta \Delta} \leq 0
$$

eventually for $t \in \mathbb{T}$, then

(2.4) $\quad \liminf _{t \rightarrow \infty} \frac{t y(t)}{h_{2}\left(t, t_{0}\right) y^{\Delta}(t)} \geq 1$.

2.9. Lemma. [5]. Suppose that $|y|^{\Delta}$ is of one sign on $\left[t_{0}, \infty\right)_{\mathbb{T}}$ and $0<\lambda<1$. Then

$$
\frac{\left(|y|^{1-\lambda}\right)^{\Delta}}{1-\lambda} \leq \frac{|y|^{\Delta}}{|y|^{\lambda}} \quad \text { on }\left[t_{0}, \infty\right)_{\mathbb{T}} \text {. }
$$

\section{Main Results}

Our first result is as follows.

3.1. Theorem. Let (i)-(v) be satisfied, and (2.1) hold. Suppose that

$$
\begin{aligned}
& \limsup _{t \rightarrow \infty} \frac{1}{a(g(t))} \int_{g(t)}^{t} q(s) h_{3}^{\beta}\left(g(s), t_{0}\right) \Delta s>1, \\
& \limsup _{t \rightarrow \infty} \int_{g(t)}^{t}\left[\frac{1}{a(u)} \int_{u}^{t}(g(t)-g(s))^{\beta} g^{\beta}(s) q(s) \Delta s\right]^{1 / \alpha} \Delta u>1,
\end{aligned}
$$

and

$$
\limsup _{t \rightarrow \infty} \frac{1}{a(l(t))} \int_{l(t)}^{t} q(s) h_{3}^{\beta}(l(t), l(s)) \Delta s>1
$$

when $\beta=\alpha$, while

$$
\begin{aligned}
& \int_{t_{0}}^{\infty} a^{-\beta / \alpha}(s) q(s) h_{3}^{\beta}\left(g(s), t_{0}\right) \Delta s=\infty, \\
& \limsup _{t \rightarrow \infty} \int_{t_{0}}^{\infty}\left[\frac{1}{a(u)} \int_{u}^{t}(t-g(s))^{\beta} g^{\beta}(s) q(s) \Delta s\right]^{1 / \alpha} \Delta u=\infty,
\end{aligned}
$$

and

$$
\int_{t_{0}}^{\infty} a^{-\beta / \alpha}(s) q(s) h_{3}^{\beta}(s, l(s)) \Delta s=\infty
$$

when $\beta<\alpha$.

Then Eq. (1.1) is oscillatory.

Proof. Let $x(t)$ be a nonoscillatory solution of equation (1.1). We may assume that $x(t)>0, x(g(t))>0$, and $x(h(t))>0$ eventually. Let $z(t)=x(t)-p(t) x(h(t))$. By Lemma 2.6, we need to consider three possible cases.

Suppose (a) holds. For any given $c_{1} \in(0,1)$ there exists $t_{1}>t_{0}$ such that

$$
z^{\Delta \Delta}(t) \geq c_{1} t z^{\Delta \Delta \Delta}(t), \quad t \in\left[t_{1}, \infty\right)_{\mathbb{T}} .
$$

From (2.4) with $y=z^{\Delta}$, for any given $c_{2}, 0<c_{2}<1$, there exists $t_{1}^{\prime}>t_{1}$ such that

$$
y(t) \geq c_{2} \frac{h_{2}\left(t, t_{0}\right)}{t} y^{\Delta}(t), \quad t \in\left[t_{1}^{\prime}, \infty\right)_{\mathbb{T}} .
$$

Combining (3.7) and (3.8) we get

$$
z^{\Delta}(t) \geq c h_{2}\left(t, t_{0}\right) z^{\Delta \Delta \Delta}(t), \quad t \in\left[t_{1}^{\prime}, \infty\right)_{\mathbb{T}}
$$

where $c:=c_{1} c_{2} \in(0,1)$ is an arbitrary constant. In view of the monotonicity of $z^{\Delta \Delta \Delta}$, it follows from (3.9) that there is a $t_{2} \geq t_{1}^{\prime}$ such that

$$
z(g(t)) \geq c h_{3}\left(g(t), t_{0}\right) z^{\Delta \Delta \Delta}(g(t)), \quad t \in\left[t_{2}, \infty\right)_{\mathbb{T}} .
$$


Using (3.10) and the fact that $x(t) \geq z(t)$ in Eq. (1.1), we obtain

$$
\left(a(t) w^{\alpha}(t)\right)^{\Delta}+c^{\beta} q(t) h_{3}^{\beta}\left(g(t), t_{0}\right) w^{\beta}(g(t)) \leq 0, \quad t \in\left[t_{2}, \infty\right)_{\mathbb{T}},
$$

where $w=z^{\Delta \Delta \Delta}$. Integrating (3.11) from $g(t)$ to $t$ leads to

$$
\begin{aligned}
a(g(t)) w^{\alpha}(g(t)) & \geq c^{\beta} \int_{g(t)}^{t} q(s) h_{3}^{\beta}\left(g(s), t_{0}\right) w^{\beta}(g(s)) \Delta s \\
& \geq c^{\beta} w^{\beta}(g(t)) \int_{g(t)}^{t} q(s) h_{3}^{\beta}\left(g(s), t_{0}\right) \Delta s
\end{aligned}
$$

and hence

$$
w^{\alpha-\beta}(g(t)) \geq \frac{c^{\beta}}{a(g(t))} \int_{g(t)}^{t} q(s) h_{3}^{\beta}\left(g(s), t_{0}\right) \Delta s
$$

which contradicts (3.1) when $\beta=\alpha$ by taking the limsup of both sides as $t \rightarrow \infty$ and then letting $c \rightarrow 1^{-}$. Let $\beta<\alpha$. Setting $v(t)=a(t) w^{\alpha}(t)$ in (3.11) and increasing the size of $t_{2}$ if necessary we have

$$
v^{\Delta}+c^{\beta} a^{-\beta / \alpha}(t) q(t) h_{3}^{\beta}\left(g(t), t_{0}\right) v^{\beta / \alpha} \leq 0, \quad t \in\left[t_{2}, \infty\right)_{\mathbb{T}}
$$

or

$$
-\frac{v^{\Delta}}{v^{\beta / \alpha}} \geq c^{\beta} a^{-\beta / \alpha}(t) q(t) h_{3}^{\beta}\left(g(t), t_{0}\right), \quad t \in\left[t_{2}, \infty\right)_{\mathbb{T}} .
$$

Integrating this inequality from $t_{2}$ to $t$ and applying Lemma 2.9 we get

$$
-\frac{v^{1-\beta / \alpha}(t)}{1-\beta / \alpha}+\frac{v^{1-\beta / \alpha}\left(t_{2}\right)}{1-\beta / \alpha} \geq-\int_{t_{2}}^{t} c^{\beta} a^{-\beta / \alpha}(s) q(s) h_{3}^{\beta}\left(g(s), t_{0}\right) \Delta s .
$$

Letting $t \rightarrow \infty$ we obtain a contradiction to (3.4).

Suppose (b) holds. For any given $k \in(0,1)$ there exists $t_{1} \geq t_{0}$ such that

(3.12) $\quad z(g(t)) \geq k g(t) z^{\Delta}(g(t)), \quad t \in\left[t_{1}, \infty\right)_{\mathbb{T}}$.

Using $x(t) \geq z(t)$ and (3.12) in Eq. (1.1) leads to

(3.13) $\quad\left(a(t)\left(y^{\Delta \Delta}(t)\right)^{\alpha}\right)^{\Delta}+k^{\beta} g^{\beta}(t) q(t) y^{\beta}(g(t)) \leq 0, \quad t \in\left[t_{1}, \infty\right)_{\mathbb{T}}$,

where $y=z^{\Delta}$. In view of $y(t)>0, y^{\Delta}(t)<0$, and $y^{\Delta \Delta}(t)>0$, we have from (3.13)

$$
y^{\Delta \Delta}(u) \geq\left[\frac{k^{\beta}}{a(u)} \int_{u}^{t} g^{\beta}(s) q(s) y^{\beta}(g(s)) \Delta s\right]^{1 / \alpha}, \quad t \geq u \geq t_{1} .
$$

Clearly,

$$
-y(g(s)) \leq \int_{g(s)}^{g(t)} y^{\Delta}(u) \Delta u \leq(g(t)-g(s)) y^{\Delta}(g(t)), \quad t \geq s \geq t_{1} .
$$

From (3.14) and (3.15) we get

$$
y^{\Delta \Delta}(u) \geq\left[\frac{k^{\beta}}{a(u)} \int_{u}^{t} g^{\beta}(s)(g(t)-g(s))^{\beta} q(s)\left(-y^{\Delta}(g(t))\right)^{\beta} \Delta s\right]^{1 / \alpha},
$$

and hence

$$
-y^{\Delta}(g(t)) \geq k^{\beta / \alpha} \int_{g(t)}^{t}\left[\frac{1}{a(u)} \int_{u}^{t} g^{\beta}(s)(g(t)-g(s))^{\beta} q(s) \Delta s\right]^{1 / \alpha} \Delta u\left(-y^{\Delta}(g(t))\right)^{\beta / \alpha}
$$

or

$$
\left(-y^{\Delta}(g(t))\right)^{1-\beta / \alpha} \geq k^{\beta / \alpha} \int_{g(t)}^{t}\left[\frac{1}{a(u)} \int_{u}^{t} g^{\beta}(s)(g(t)-g(s))^{\beta} q(s) \Delta s\right]^{1 / \alpha} \Delta u
$$


which as in case (a) results in a contradiction with (3.2) when $\beta=\alpha$. Let $\beta<\alpha$. From (3.15) we have

$$
-y(g(s)) \leq(t-g(s)) y^{\Delta}(t), \quad t \geq s \geq t_{1} .
$$

Using this inequality in (3.14) and setting $v=-y^{\Delta}$ we have

$$
-\frac{v^{\Delta}(u)}{v^{\beta / \alpha}(u)} \geq\left[\frac{k^{\beta}}{a(u)} \int_{u}^{t} g^{\beta}(s)(t-q(s))^{\beta} \Delta s\right]^{1 / \alpha}, \quad t \geq u \geq t_{1} .
$$

The rest is similar to that of case (a) above and hence is omitted.

Suppose (c) holds. It follows that

$$
x(g(t)) \geq y\left(h^{-1} \circ g(t)\right), \quad t \in\left[t_{1}, \infty\right)_{\mathbb{T}},
$$

where $y=-z$. By Eq. (1.1), we may write that

$$
\left(a(t)\left(y^{\Delta \Delta \Delta}\right)^{\alpha}\right)^{\Delta} \geq q(t) y^{\beta}(l(t)), \quad t \in\left[t_{1}, \infty\right)_{\mathbb{T}} .
$$

In view of

$$
y^{\Delta}(t)<0, \quad y^{\Delta \Delta}(t)>0, \quad y^{\Delta \Delta \Delta}(t)<0, \quad t \in\left[t_{1}, \infty\right)_{\mathbb{T}}
$$

and

we see that

$$
y^{\Delta \Delta}(s) \geq h_{1}(t, s)\left(-y^{\Delta \Delta \Delta}(t)\right)
$$

$$
y(s) \geq h_{3}(t, s)\left(-y^{\Delta \Delta \Delta}(l(t))\right), \quad t \geq s \geq t_{1} .
$$

Replacing $s$ by $l(s)$ and $t$ by $l(t)$ in the above inequality leads to

$$
y(l(s)) \geq h_{3}(l(t), l(s))\left(-y^{\Delta \Delta \Delta}(t)\right), \quad l(t) \geq l(s) \geq t_{1} .
$$

Integrating (3.16)) and using (3.17) we obtain

$$
a(l(t)) w^{\alpha}(l(t)) \geq \int_{l(t)}^{t} q(s) h_{3}^{\beta}(l(t), l(s)) w^{\beta}(l(s)) \Delta s, \quad w:=-y^{\Delta \Delta \Delta},
$$

and hence

$$
w^{\alpha-\beta}(l(t)) \geq \frac{1}{a(l(t))} \int_{l(t)}^{t} q(s) h_{3}^{\beta}(l(t), l(s)) \Delta s .
$$

This inequality contradicts (3.3) when $\beta=\alpha$ by taking the limsup of both sides as $t \rightarrow \infty$.

It remains to consider $\beta<\alpha$. Set $v=a(t) w^{\alpha}$ and $w=-y^{\Delta \Delta \Delta}$ in (3.16). Then we have

$$
-v^{\Delta}(t) \geq q(t) y^{\beta}(l(t)), \quad t \in\left[t_{1}, \infty\right)_{\mathbb{T}} .
$$

As in (3.17) we can obtain

$$
y(l(t)) \geq h_{3}(t, l(t)) w(t), \quad t \geq s \geq t_{1} .
$$

Thus

$$
-v^{\Delta}(t) \geq q(t) h_{3}^{\beta}(t, l(t)) w^{\beta}(t), \quad t \in\left[t_{1}, \infty\right)_{\mathbb{T}} .
$$

The remainder is similar to that of cases (a) and (b) and hence is omitted.

3.2. Theorem. Let (i)-(v) be satisfied, and (2.1) hold. In addition to (3.3) and (3.6), suppose that for $k \in\{1,3\}$,

$$
\limsup _{t \rightarrow \infty} h_{k}^{\beta}\left(g(t), t_{0}\right) h_{3-k}^{\beta}(t, g(t)) \frac{1}{a(t)} \int_{t}^{\infty} q(s) \Delta s>1 \quad \text { when } \beta=\alpha
$$

and

$$
\int_{t_{0}}^{\infty} h_{k}^{\beta}\left(g(t), t_{0}\right) h_{3-k}^{\beta}(t, g(t)) a^{-\beta / \alpha}(t) q(t) \Delta t=\infty \quad \text { when } \beta<\alpha
$$


Then Eq. (1.1) is oscillatory.

Proof. Let $x(t)$ be a nonoscillatory solution of equation (1.1), say $x(t)>0, x(g(t))>0$, and $x(h(t))>0$ for $t \in\left[t_{0}, \infty\right)_{\mathbb{T}}$, and let $z(t)=x(t)-p(t) x(h(t))$. As in the proof of Theorem 3.1 we need to distinguish three possible cases.

We may claim that

(3.20) $\quad z(g(t)) \geq h_{k}\left(g(t), t_{1}\right) h_{3-k}(t, g(t)) z^{\Delta \Delta \Delta}(t) \quad$ for $k \in\{1,3\}$ and $g(t) \geq t_{1}$

when (a) and (b) are satisfied.

Indeed, if (a) holds, then integrating the inequality

$$
z^{\Delta \Delta}(v) \geq z^{\Delta \Delta}(v)-z^{\Delta \Delta}\left(u_{1}\right)=\int_{u_{1}}^{v} z^{\Delta \Delta \Delta}(s) \Delta s \geq h_{1}\left(v, u_{1}\right) z^{\Delta \Delta \Delta}(v)
$$

twice leads to

$$
z(v) \geq h_{3}\left(v, u_{1}\right) z^{\Delta \Delta \Delta}(v), \quad v \geq u_{1} \geq t_{1} .
$$

Also

$$
z^{\Delta \Delta}(v) \geq z^{\Delta \Delta}(v)-z^{\Delta \Delta}(u)=\int_{u}^{v} z^{\Delta \Delta \Delta}(s) \Delta s \geq h_{1}(v, u) z^{\Delta \Delta \Delta}(v)
$$

gives

(3.22) $\quad z^{\Delta}(v) \geq h_{2}(v, u) z^{\Delta \Delta \Delta}(v)$.

Putting $v=g(t) \geq t_{1}$ and $v=t$ into (3.22), we have

$$
z^{\Delta}(g(t)) \geq h_{2}(t, g(t)) z^{\Delta \Delta \Delta}(t),
$$

i.e.,

(3.23) $\quad z^{\Delta_{k}}(g(t)) \geq h_{3-k}(t, g(t)) z^{\Delta \Delta \Delta}(t) \quad$ for $k \in\{1,3\}$ and $g(t) \geq t_{1}$.

If (b) holds, then

$$
z(v) \geq z(v)-z\left(u_{1}\right)=\int_{u_{1}}^{v} z^{\Delta}(s) \Delta s \geq h_{1}\left(v, u_{1}\right) z^{\Delta}(v) .
$$

Clearly, (3.21) and (3.24) can be written at once

$$
z(v) \geq h_{k}\left(v, u_{1}\right) z^{\Delta^{k}}(v) \text { for } k \in\{1,3\} \text { and } v \geq u_{1} \geq t_{1},
$$

and hence

(3.25) $\quad z(g(t)) \geq h_{k}\left(g(t), t_{1}\right) z^{\Delta^{k}}(g(t)) \quad$ for $k \in\{1,3\}$ and $g(t) \geq t_{1}$ when $g(t) \geq t_{1}$. From (3.23) and (3.25) the claim follows.

Now by using $x(t) \geq z(t)$ in (1.1) and integrating the resulting inequality from $t$ to $u$ and then letting $u \rightarrow \infty$, we have

$$
w^{\alpha}(t) \geq\left[\frac{1}{a(t)} \int_{t}^{\infty} q(s) \Delta s\right] z^{\beta}(g(t)), \quad t \geq t_{1},
$$

where $w=z^{\Delta \Delta \Delta}$. Using (3.20) in (3.26) leads to

$$
w^{\alpha-\beta}(t) \geq h_{k}\left(g(t), t_{1}\right) h_{3-k}(t, g(t))\left[\frac{1}{a(t)} \int_{t}^{\infty} q(s) \Delta s\right], \quad t \geq t_{1},
$$

which contradicts (3.18) when $\beta=\alpha$. If $\beta<\alpha$, we first write from (1.1) that

$$
\left(a(t) w^{\alpha}(t)\right)^{\Delta}+q(t) z^{\beta}(g(t)) \leq 0, \quad t \in\left[t_{0}, \infty\right)_{\mathbb{T}} .
$$

Using (3.20) and $v=a(t) w^{\alpha}$ in (3.27), we have

$$
-v^{\Delta} \geq h_{k}^{\beta}\left(g(t), t_{1}\right) h_{3-k}^{\beta}(t, g(t)) a^{-\beta / \alpha}(t) q(t) v^{\beta / \alpha}, \quad t \in\left[t_{0}, \infty\right)_{\mathbb{T}} .
$$


The rest of the proof is similar to that above and hence is omitted.

Finally, we need to consider the case (c). Since the proof for this case is similar to that of Theorem 3.1-case (c), we omit the details.

In case (2.3) holds, we have the following similar theorems.

3.3. Theorem. Let (i) $-(v)$ be satisfied, and (2.3) hold. Suppose that

$$
\limsup _{t \rightarrow \infty} \frac{1}{a(g(t))} \int_{g(t)}^{t} q(s)[1+p(s)]^{\beta} h_{3}^{\beta}\left(g(s) t_{0}\right) \Delta s>1,
$$

and

$$
\limsup _{t \rightarrow \infty} \int_{g(t)}^{t}\left[\frac{1}{a(u)} \int_{u}^{t}(g(t)-g(s))^{\beta}[1+p(s)]^{\beta} q(s) \Delta s\right]^{1 / \alpha} \Delta u>1
$$

when $\beta=\alpha$, while

$$
\int_{t_{0}}^{\infty} a^{-\beta / \alpha}(s)[1+p(s)]^{\beta} q(s) h_{3}^{\beta}\left(g(s), t_{0}\right) \Delta s=\infty
$$

and

$$
\limsup _{t \rightarrow \infty} \int_{t_{0}}^{\infty}\left[\frac{1}{a(u)} \int_{u}^{t}(t-g(s))^{\beta} g^{\beta}(s)[1+p(s)]^{\beta} q(s) \Delta s\right]^{1 / \alpha} \Delta u=\infty
$$

when $\beta<\alpha$.

Then Eq. (1.1) is oscillatory.

Proof. Let $x(t)$ be a nonoscillatory solution of (1.1), say $x(t)>0, x(g(t))>0$, and $x(\tau(t))>0$ for $t \in\left[t_{0}, \infty\right)_{\mathbb{T}}$. It is clear that

$$
z(t)=x(t)-p(t) x(h(t)) \geq x(t) .
$$

By Lemma $2.7, z^{\Delta}(t)$ is eventually positive, and so

$$
x(t)=z(t)+p(t) x(h(t)) \geq z(t)+p(t) z(h(t)) \geq[1+p(t)] z(t)
$$

for $t \in\left[t_{1}, \infty\right)_{\mathbb{T}}$ for some $t_{1} \geq t_{0}$. In view of this inequality and Eq. (1.1) we get

$$
\left(a(t)\left(z^{\Delta \Delta \Delta}(t)\right)^{\alpha}\right)^{\Delta}+q(t)[1+p(g(t))]^{\beta} z^{\beta}(g(t)) \leq 0, \quad t \in\left[t_{0}, \infty\right)_{\mathbb{T}} .
$$

The remainder of the proof proceeds from (3.32) similarly as in the cases (a) and (b) of that of Theorem 3.1.

3.4. Theorem. Let (i)-(v) be satisfied, and (2.3) hold. Suppose that for $k \in\{1,3\}$,

$$
\limsup _{t \rightarrow \infty} h_{k}^{\beta}\left(g(t), t_{0}\right) h_{3-k}^{\beta}(t, g(t)) \frac{1}{a(t)} \int_{t}^{\infty}[1+p(g(s))]^{\beta} q(s) \Delta s>1 \text { when } \beta=\alpha
$$

and

$$
\int_{t_{0}}^{\infty} h_{k}^{\beta}\left(g(t), t_{0}\right) h_{3-k}^{\beta}(t, g(t)) a^{-\beta / \alpha}(t)[1+p(g(t))]^{\beta} q(t) \Delta t=\infty \text { when } \beta<\alpha .
$$

Then Eq. (1.1) is oscillatory.

The results of this paper are presented in a form which is essentially new and of high degree of generality. We note that the obtained results when $\beta=\alpha$ (half-linear case) are not applicable to equations of type (1.1) with $g(t)=t$. This means that the delays generate oscillation. 
It is possible to formulate the corresponding theorems and give illustrative examples for special time scales such as $\mathbb{T}=\mathbb{R}, \mathbb{T}=\mathbb{Z}, \mathbb{T}=h \mathbb{Z}$ with $h>0, \mathbb{T}=q^{\mathbb{N}}$ with $q>1$, $\mathbb{T}=\mathbb{N}^{2}$. The details are left to the reader.

It would be of interest to study the oscillatory behaviour of all solutions of (1.1) when $\beta>\alpha$ (super half-linear case) or $p(t) \leq-1$ or $p(t)>1$.

\section{References}

[1] D. R. Anderson and A. Zafer, Interval criteria for second-order super-half-linear functional dynamic equations with delay and advance arguments. J. Difference Equ. Appl. 16 (2010), 917-930.

[2] M. Bohner, G. Sh. Guseinov, Improper integrals on time scales. Special issue: dynamic equations on time scales. Dynam. Systems Appl. 12 (2003), 45-65.

[3] M. Bohner and A. Peterson, Dynamic Equations on Time-Scales : An Introduction with Applications, Birkhauser, Boston 2001.

[4] M. Bohner and R. P. Agarwal, Basic calculus on time scales and some of its applications. Result Math. 35 (1999), 3-22

[5] L. Erbe, A. Peterson, and S. Saker, Hille and Nehari type criteria for third order dynamic equations, J. Math. Anal. Appl. 333 (2007), 505-522.

[6] L. Erbe, R. Mert, A. Peterson, and A, Zafer, Oscillation of even order nonlinear delay dynamic equations on time scales. In: Czech Math J

[7] S.R. Grace, M. Bohner, and R.P. Agarwal, On the oscillation of second order half linear dynamic equations, J. Diff. Eqns. Appl. 15 (2009), 451-460.

[8] S. R. Grace, M. Bohner, and S. Sun, Oscillation of fourth-order dynamic equations, Hacet. J. Math. Stat. 39 (4) (2010), 545-553

[9] G. Sh. Guseionov, Integration on time scales. J. Math. Anal. Appl. 285 (2003), 107?127.

[10] Z. Han, T. Li, S. Sun, and C. Zhang, Oscillation behaviour of third order neutral EmdenFowler delay dynamic equations on time-scales, Adv. Diff. Eqns. 2010 (2010), Article ID.586312, 23 pages.

[11] Z. Han, S. Sun and B. Shi, Oscillation criteria for a class of second order Emden-Fowler delay dynamic equations on time-scales, J. Math. Anal. Appl. 334 (2007), 847-858.

[12] S. Hilger, Ein Maßkettenkalkül mit A nwendung auf Zentrumsmannigfaltigkeiten, $\mathrm{PhD}$ thesis, Universität Würzburg, 1988.

[13] T. Li, E. Thandapani, and S. Tang, Oscillation theorems for fourth-order delay dynamic equations on time scales, Bull. Math. Anal. Appl. 3 (2011), 190-199.

[14] A. Zafer, On oscillation and nonoscillation of second-order dynamic equations. Appl. Math. Lett. 22 (2009), no. 1, 136-141, 
